\title{
Olfactory Impairment Predicts Brain Atrophy in Parkinson's Disease
}

\author{
Elise Wattendorf, ${ }^{1,2}$ Antje Welge-Lüssen, ${ }^{2}$ Klaus Fiedler, ${ }^{1}$ Deniz Bilecen, ${ }^{3}$ Markus Wolfensberger, ${ }^{2}$ Peter Fuhr, ${ }^{4}$ \\ Thomas Hummel, ${ }^{5}$ and Birgit Westermann ${ }^{1}$ \\ ${ }^{1}$ Department of Neurosurgery, ${ }^{2}$ Department of Otorhinolaryngology, ${ }^{3}$ Institute of Radiology, and ${ }^{4}$ Department of Neurology, University Hospital, \\ University of Basel, CH-4031 Basel, Switzerland, and ${ }^{5}$ Smell and Taste Clinic, University of Dresden Medical School, D-01037 Dresden, Germany
}

Olfactory dysfunction is a frequent nonmotor symptom in idiopathic Parkinson's disease (PD) and may be considered as an early clinical feature of the disease preceding motor symptoms by years. According to recent neuropathological staging concepts, impaired olfaction is assumed to indicate an early pathological process and might be associated with structural changes in the brain. A morphometric analysis of magnetic resonance images [voxel-based morphometry (VBM)] was used to investigate gray matter atrophy related to psychophysically measured scores of olfactory function in early PD patients $(n=15$, median Hoehn and Yahr stage 1.5), moderately advanced PD patients $(n=12$, median Hoehn and Yahr stage 2.5$)$, and age-matched healthy controls $(n=17)$. In PD patients, but not in controls, cortical atrophy in olfactory-related brain regions correlated specifically with olfactory dysfunction. Positive correlations between olfactory performance and gray matter volume were observed in the right piriform cortex in early PD patients and in the right amygdala in moderately advanced patients. The results provided first evidence that olfactory dysfunction in PD is related to atrophy in olfactory-eloquent regions of the limbic and paralimbic cortex. In addition, olfactory-correlated atrophy in these brain regions is consistent with the assumption that olfactory impairment as an early symptom of PD is likely to be associated with extranigral pathology.

\section{Introduction}

It is now widely accepted that early nonmotor signs indicate preclinical stages of Parkinson's disease (PD) before the onset of motor symptoms. Consistent with this clinical observation, Braak's neuropathological staging concept (Braak et al., 2003) proposes that important pathological processes during the presymptomatic phase may occur already in other, primarily non-motorrelated brain regions. While cardinal motor symptoms in PD are closely related to a severe loss of dopaminergic cells in the nigrostriatal pathway, early clinical features are more likely to be associated with extranigral pathology. A prevalent symptom at early stages of PD is olfactory impairment (Ansari and Johnson, 1975). Results from postmortem studies revealed pathological changes (Lewy body formation) in the olfactory bulb (Huisman et al., 2008) but also in other brain regions related to olfaction, such as the anterior olfactory nucleus (Pearce et al., 1995), the piriform cortex (Braak et al., 2003; Silveira-Moriyama et al., 2009), the amygdaloid complex (Harding et al., 2002; Braak et al., 2003), the entorhinal cortex, and the hippocampal formation (Braak et al., 2003). Using functional magnetic resonance imaging in PD patients, our previous findings indicated altered neuronal activity in the amygdaloid complex and hippocampal formation during olfactory stimulation (Westermann et al., 2008; Welge-Lüssen et al., 2009). On this basis, we hypothesized that pathophysiology of

\footnotetext{
Received April 22, 2009; revised 0ct. 2, 2009; accepted 0ct. 30, 2009.

This study was supported by a grant of the Swiss National Science Foundation (Grant 3100-068282).

Correspondence should be addressed to Dr. Birgit Westermann, Neurosurgical University Clinic, University Hos-

pital, University of Basel, Spitalstrasse 21, CH-4031 Basel, Switzerland. E-mail: birgit.westermann@unibas.ch. DOI:10.1523/JNEUROSCI.1909-09.2009

Copyright $\odot 2009$ Society for Neuroscience $\quad$ 0270-6474/09/2915410-04\$15.00/0
}

olfaction in PD is associated with structural abnormalities, for instance with regional atrophy in brain regions involved in olfaction. For in vivo studying of structural brain anatomy, voxelbased morphometry (VBM) has been proven to correlate clinical features with gray matter changes in $\mathrm{PD}$, such as executive and visuospatial impairment (Nagano-Saito et al., 2005) and depression (Feldmann et al., 2008). However, olfactory dysfunction as an early symptom of PD has not yet been investigated with respect to structural changes in the brain. Aiming at this question, we performed a morphometric analysis of magnetic resonance images (VBM) to identify gray matter atrophy related to olfactory dysfunction in three study groups: early PD patients, moderately advanced PD patients, and healthy controls.

\section{Materials and Methods}

Subjects. We investigated 27 patients with PD diagnosed according to the UK PD Society Brain Bank diagnostic criteria (Gibb and Lees, 1988) and 17 age-matched healthy controls ( 9 men, mean age: 63.1 years, range: 52-72 years). None of the control subjects had a history or signs of major neurological disorders. Written informed consent was obtained from all participants. The study was approved by the Ethics Committee of the University Hospital of Basel, Switzerland. According to the Hoehn and Yahr scale (H\&Y), early PD patients $(n=15,11$ men, mean age: 60.3 years, range: $44-71$ years) had a median score of 1.5 , while moderately advanced PD patients ( $n=12,6$ men, mean age: 61.7 years, range: $46-69$ years) had a median score of 2.5 (Hoehn and Yahr, 1967). The median Unified Parkinson's Disease Rating Scale score was 26 (range: 4-41) in early patients and 30 (range: 18-60) in moderately advanced patients (Fahn and Elton, 1987). The median duration of disease was 3.2 and 8.8 years, respectively. All subjects were nondemented [Mini-Mental State Examination (MMSE) (Folstein et al., 1975), MMSE score >28] and did 


\section{Early PD patients \\ Cortical atrophy correlated with olfactory performance}

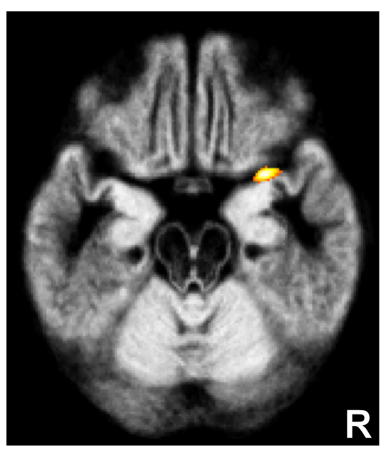

a

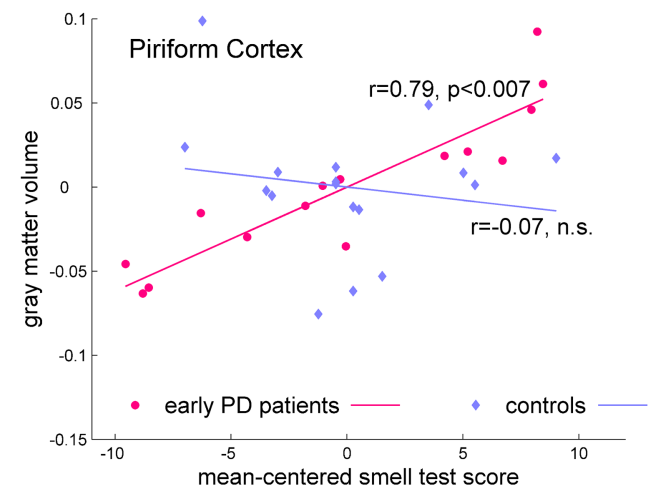

$1 \times 1 \times 1 \mathrm{~mm})$. VBM has been applied to detect alterations in gray matter volume that are associated with olfactory performance in olfactory-related regions of the limbic and paralimbic cortex in PD patients and normal healthy controls. Data analysis was performed using VBM5 toolbox (http://dbm.neuro. uni-jena.de/vbm/, version 1.18) for Statistical Parametric Mapping (Friston et al., 1995) (SPM5). The unified segmentation approach uses prior tissue maps to estimate the probability of each voxel belonging to the respective tissue class, i.e., gray and white matter (Ashburner and Friston, 2005). However, tissue priors derived from young, healthy subjects could introduce a segmentation bias in our specific study groups. To avoid such bias, segmentation based on voxel intensity alone has been applied (Gaser et al., 2007). The resulting tissue maps were modulated with the Jacobian determinants of the deformation parameters obtained by normalization to the MNI (Montreal Neurological Institute) standard space. A customized template consisting of all subjects in our study was created to improve the quality of normalization (Wilke et al., 2008). Finally, images were smoothed with an isotropic Gaussian kernel of $8 \mathrm{~mm}$.

The present analysis was designed to assess cortical atrophy that correlated with olfactory performance (TDI score). Changes of gray matter volume dependent on TDI scores were assessed in a multiple regression analysis including total gray matter volume as nuisance covariate. In PD patients, this analysis was additionally corrected for the H\&Y score and disease duration. Based on our a priori hypothesis, a statistical threshold of $p<0.005$ (uncorrected) with a cluster extent of 800 voxel was used. The cluster extent threshold has been corrected for anisotropy of smoothness (Hayasaka et al., 2004). The correlation coefficients were computed as average across all voxels of each cluster in the respective regions. Group comparisons in correlations were considered significant at $p<0.005$ (uncorrected). Correlation maxima were reported as $Z$-scores and not exhibit significant signs of depression [Beck Depression Inventory (BDI) (Beck et al., 1961), BDI score < 15].

Olfactory testing. Olfactory function was assessed birhinally using the standardized "Sniffin' Sticks" test battery (Burghart) that included nonverbal odor threshold (T), nonverbal discrimination (D), and verbal identification (I) tests (Hummel et al., 1997; Kobal et al., 2000).

The sum of scores obtained from the three subtests threshold, discrimination, and identification gives a composite measure of olfactory function, i.e., the TDI-score (see supplemental text 1, available at www. jneurosci.org as supplemental material). Based on the TDI score, olfactory function is classified as normosmic (TDI >30), hyposmic (TDI $16-30)$, and functionally anosmic (TDI $\leq 15$ ). On average, early and moderately advanced PD patients were hyposmic as indicated by mean scores of 21.8 (range: 12.3-30.3) and 21.3 (range: 15.0-32.5), respectively. The mean olfactory scores did not differ between both PD groups $[p=0.85$, not significant (n.s.) $]$. Healthy controls were, on average, normosmic (mean score: 33.9, range: $27.0-43.0$ ) and had a better olfactory performance than early and advanced PD patients (for both $p<$ $0.00001)$.

Data acquisition and analysis. Images were acquired on a $1.5 \mathrm{~T}$ Siemens Sonata MR scanner (Siemens) using a T1-weighted 3D magnetization prepared gradient rapid acquisition gradient echo (MPRAGE) sequence (echo time: $3.68 \mathrm{~ms}$, repetition time: $1900 \mathrm{~ms}$, flip angle: $10^{\circ}$, voxel size:

coordinates in the MNI space. The investigator was blinded with regard to group assignment and scores from clinical examination including motor and olfactory scores.

\section{Results}

In PD patients, but not in controls, gray matter volume correlated positively with increasing scores of olfactory function as obtained by psychophysical measurements. The early PD group showed a significant correlation of gray matter volume and olfactory performance in a primary olfactory region, namely the right piriform cortex (Fig. 1a). Moderately advanced patients exhibited a correlative effect in the right amygdaloid complex (Fig. 1b), a region corresponding to secondary olfactory projection zones. It has to be noted that the linear increase of gray matter volume with better olfactory function was most significant in olfactory-related brain regions in both PD groups. The cluster-level inference indicated that the clusters in the piriform cortex and amygdala were significant at $p<0.007$ and $p<0.003$, respectively (Fig. $1 c, d$ ). In contrast, controls showed no significant correlation in these brain regions (Fig. 1c,d). Effects of olfactory-correlated atrophy 
in each of the PD groups were confirmed by group comparisons. When compared to controls, early PD patients showed a stronger correlative effect in the right piriform cortex $(28,6,-19 ; p<$ $0.002, z=2.96)$ and advanced patients in the right amygdala (23, $-1,-23 ; p<0.005, z=2.76$ ). These effects were also specific for each of the PD groups. In early PD patients, correlation was significantly higher in the right piriform cortex than in advanced patients $(28,7,-20 ; p<0.004 ; z=2.65)$. Vice versa, in advanced patients, a trend for higher correlation was observed in the right amygdala $(19,0,-23, p<0.02, z=2.0)$.

The comparison on total intracranial volume (TIV) and gray matter volume (GMV) did not show any difference between all groups studied (supplemental Fig. 1, available at www.jneurosci. org as supplemental material).

\section{Discussion}

In this study, VBM was applied to detect gray matter atrophy that correlated with scores of olfactory function in PD patients and controls. Our results suggest that olfactory dysfunction in PD is associated with atrophy in olfactory-eloquent regions of the limbic and paralimbic cortex and might therefore not be restricted to deficient sensory transmission in the olfactory bulb (Huisman et al., 2008). Further analysis showed that olfactory-related atrophy is not a consequence of generalized brain atrophy but seems to be rather a regional effect; total intracranial and gray matter volumes did not differ between all groups. Moreover, these local correlative effects were confirmed as specific features of PD by group comparisons indicating that the correlation between olfactory scores and gray matter atrophy is significantly higher in each of the PD groups than in normal controls.

The positive correlation of better olfactory performance with increasing gray matter volume in early PD patients implies that the piriform cortex contributes critically to normal olfactory function. However, it is noteworthy that the function of the piriform cortex extends beyond merely unimodal sensory processing. Its involvement in complex aspects of odor information processing, such as olfactory associative learning, indicates that this region also plays a role in establishing cross-modal associations (Gottfried et al., 2002, 2004; Boyle et al., 2007; Hummel et al., 2009). Access to cross-modal associations is also necessary for successful retrieval of chemosensory information when odors are identified with the help of verbal descriptors. Verbal odor identification was part of the olfactory assessment in the present study. Our finding of an olfactory-correlated atrophy in the piriform cortex partly provides an explanation for deficits of PD patients on this task (Doty et al., 1988, 1992).

In moderately advanced PD patients, olfactory performance correlated positively with gray matter volume in the amygdala. This finding is consistent with postmortem studies suggesting that substantial cell loss accompanied by high densities of Lewy bodies in this brain region contributes to impaired olfaction (Harding et al., 2002). However, our results imply that olfactory dysfunction is associated with degenerative changes of the amygdala at earlier stages of PD and not only at final stages in which the disease has reached its fully clinical extent. Olfactory perception involves processes in the amygdala to evaluate the affective value of stimuli (Zald and Pardo, 1997; Winston et al., 2005). Thus, olfactory-correlated atrophy in this brain region could indicate that PD patients have difficulties to assess the emotional valence of olfactory stimuli. Indirect support for this interpretation comes from our previous fMRI study that showed reduced activity in the amygdala in PD patients during olfactory stimulation (Westermann et al., 2008).
The reason for this group-specific atrophy related to olfaction in both PD groups remains speculative. The comparison of olfactory-correlated atrophy between the PD groups showed a significantly higher correlation in the piriform cortex in early PD patients than in advanced patients. In advanced patients, a trend for higher correlation could be demonstrated in the amygdala. The observed correlative effects are independent from disease severity (H\&Y score) and disease duration as both variables were included as covariates in the regression analysis. However, it has to be considered that both parameters are related to the symptomatic phase of PD, i.e., after onset of cardinal motor symptoms. In contrast, olfactory impairment as an early symptom indicates the presymptomatic (nonmotor) phase of the disease. Possibly, our finding of a group-specific correlation between cortical atrophy and olfactory impairment suggests that extranigral pathology has progressed in regions of the olfactory cortex. This interpretation is consistent with the previously mentioned spreading of PD-related brain lesions from the anterior olfactory nucleus (stage 1) to the piriform cortex and different subnuclei of the amygdala (stage 3/4) (Braak et al., 2003). In earlier affected brain areas, it might therefore no longer be possible to show a correlation at later stages of the disease. Thus, a stronger atrophy of the piriform cortex could explain the missing correlative effect in the moderately advanced PD group.

In addition, a correlation of olfactory ability with atrophy in regions of the nigrostriatal system in either patient group was absent emphasizing the role of extranigral pathology on impaired olfaction in PD.

Summarizing, the results provide first evidence that olfactory impairment is a valuable biomarker to reveal progressive cortical atrophy during early and moderately advanced stages of PD. Concerning disease progression, olfactory symptoms in PD indicate a pathological process that seems to be present in parallel to the depletion of the nigrostriatal dopaminergic pathway. In view of an early, extranigral pathology, morphometric data together with the assessment of olfactory function could be relevant to identify patients in presymptomatic stages of PD.

\section{References}

Ansari KA, Johnson A (1975) Olfactory function in patients with Parkinson's disease. J Chronic Dis 28:493-497.

Ashburner J, Friston KJ (2005) Unified segmentation. Neuroimage 26:839-851.

Beck AT, Ward CH, Mendelson M, Mock J, Erbaugh J (1961) An inventory for measuring depression. Arch Gen Psychiatry 4:561-571.

Boyle JA, Frasnelli J, Gerber J, Heinke M, Hummel T (2007) Cross-modal integration of intranasal stimuli: A functional magnetic resonance imaging study. Neuroscience 149:223-231.

Braak H, Del Tredici K, Rüb U, De Vos RAI, Jansen Steur ENH, Braak E (2003) Staging of brain pathology related to sporadic Parkinson's disease. Neurobiol Aging 24:197-211.

Doty RL, Deems DA, Stellar S (1988) Olfactory dysfunction in parkinsonism-a general deficit unrelated to neurologic signs, disease stage, or disease duration. Neurology 38:1237-1244.

Doty RL, Stern MB, Pfeiffer C, Gollomp SM, Hurtig HI (1992) Bilateral olfactory dysfunction in early stage treated and untreated idiopathic Parkinson's disease. J Neurol Neurosurg Psychiatry 55:138-142.

Fahn S, Elton RL (1987) Unified Parkinson's disease rating scale. In: Recent developments in Parkinson's disease (Fahn S, Marsden CD, Calne D, Goldstein M, eds), pp 153-164. Florham Park, NJ: Macmillan Health Care Information.

Feldmann A, Illes Z, Kosztolanyi P, Illes E, Mike A, Kover F, Balas I, Kovacs N, Nagy F (2008) Morphometric changes of gray matter in Parkinson's disease with depression: a voxel-based morphometry study. Mov Disord 23:42-46.

Folstein MF, Folstein SE, McHugh PR (1975) Mini-mental state - practical 
method for grading cognitive state of patients for clinician. J Psychiatr Res 12:189-198.

Friston KJ, Holmes AP, Worsley KJ, Poline JB, Frith CD, Frackowiak RS (1995) Statistical parametric maps in functional imaging: a general linear approach. Hum Brain Mapp 2:189-210.

Gaser C, Altaye M, Wilke M, Holland SK (2007) Unified segmentation without tissue priors. Neuroimage 36[Suppl 1]:S68.

Gibb WRG, Lees AJ (1988) The relevance of the Lewy body to the pathogenesis of idiopathic Parkinson's disease. J Neurol Neurosurg Psychiatry 51:745-752.

Gottfried JA, O'Doherty J, Dolan RJ (2002) Appetitive and aversive olfactory learning in humans studied using event-related functional magnetic resonance imaging. J Neurosci 22:10829-10837.

Gottfried JA, Smith APR, Rugg MD, Dolan RJ (2004) Remembrance of odors past: human olfactory cortex in cross-modal recognition memory. Neuron 42:687-695.

Harding AJ, Stimson E, Henderson JM, Halliday GM (2002) Clinical correlates of selective pathology in the amygdala of patients with Parkinson's disease. Brain 125:2431-2445.

Hayasaka S, Phan KL, Liberzon I, Worsley KJ, Nichols TE (2004) Nonstationary cluster-size inference with random field and permutation methods. Neuroimage 22:676-687.

Hoehn MM, Yahr MD (1967) Parkinsonism — onset progression and mortality. Neurology 17:427-442.

Huisman E, Uylings HBM, Hoogland PV (2008) Gender-related changes in increase of dopaminergic neurons in the olfactory bulb of Parkinson's disease patients. Mov Disord 23:1407-1413.

Hummel T, Oehme L, van den Hoff J, Gerber J, Heinke M, Boyle JA, Beuthien-Baumann B (2009) PET-based investigation of cerebral activation following intranasal trigeminal stimulation. Hum Brain Mapp 30:1100-1104.

Hummel T, Sekinger B, Wolf SR, Pauli E, Kobal G (1997) 'Sniffin' sticks': olfactory performance assessed by the combined testing of odor identifi- cation, odor discrimination and olfactory threshold. Chem Senses 22:39-52.

Kobal G, Klimek L, Wolfensberger M, Gudziol H, Temmel A, Owen CM, Seeber H, Pauli E, Hummel T (2000) Multicenter investigation of 1,036 subjects using a standardized method for the assessment of olfactory function combining tests of odor identification, odor discrimination, and olfactory thresholds. Eur Arch Otorhinolaryngol 257:205-211.

Nagano-Saito A, Washimi Y, Arahata Y, Kachi T, Lerch JP, Evans AC, Dagher A, Ito K (2005) Cerebral atrophy and its relation to cognitive impairment in Parkinson disease. Neurology 64:224-229.

Pearce RKB, Hawkes CH, Daniel SE (1995) The anterior olfactory nucleus in Parkinson's disease. Mov Disord 10:283-287.

Silveira-Moriyama L, Holton JL, Kingsbury A, Ayling H, Petrie A, Sterlacci W, Poewe W, Maier H, Lees AJ, Revesz T (2009) Regional differences in the severity of Lewy body pathology across the olfactory cortex. Neurosci Lett 453:77-80.

Welge-Lüssen A, Wattendorf E, Schwerdtfeger U, Fuhr P, Bilecen D, Hummel T, Westermann B (2009) Olfactory induced brain activity in Parkinson's disease relates to the expression of event-related potentials-an fMRI study. Neuroscience 162:537-543.

Westermann B, Wattendorf E, Schwerdtfeger U, Husner A, Fuhr P, Gratzl O, Hummel T, Bilecen D, Welge-Lüssen A (2008) Functional imaging of the cerebral olfactory system in patients with Parkinson's disease. J Neurol Neurosurg Psychiatry 79:19-24.

Wilke M, Holland SK, Altaye M, Gaser C (2008) Template-O-Matic: a toolbox for creating customized pediatric templates. Neuroimage 41:903-913.

Winston JS, Gottfried JA, Kilner JM, Dolan RJ (2005) Integrated neural representations of odor intensity and affective valence in human amygdala. J Neurosci 25:8903-8907.

Zald DH, Pardo JV (1997) Emotion, olfaction, and the human amygdala: amygdala activation during aversive olfactory stimulation. Proc Natl Acad Sci U S A 94:4119-4124. 